\title{
Mesure du dépôt de plutonium 239 atmosphérique sur les feuilles de Casuarina equisetifolia : comparaison entre Mururoa et l'ensemble de la zone intertropicale
}

\author{
H. TYMEN *, C. BOURNIGAL **, M. CAMOIT **, P. GERASIMO ***,
} R. DUCOUSSO *

(Manuscrit reçu le 9 avril 1990)

RÉSUMÉ Le Casuarina equisetifolia possède un feuillage qui ressemble à celui des conifères. De ce fait, il présente un bon rendement de captation des aérosols atmosphériques. II est très répandu dans la zone intertropicale. Nous avons pu ainsi comparer le plutonium 239 de l'atmosphère des sites expérimentaux de Mururoa et de Fangataufa à celui de l'atmosphère du reste de la Polynésie française et de différents autres points de la zone intertropicale. Aucune différence significative n'a pu être mise en évidence.

ABSTRACT Casuarina equisetifolia is a pine-like leaves tree growing all around the tropical area. Consequently, atmospheric aerosols are very efficiently retained by it. ${ }^{239} \mathrm{Pu}$ retention by Casuarina equisetifolia leaves was measured on samples from the two french nuclear test sites (Mururoa and Fangataufa), other french Polynesia archipelagoes and various tropical countries. The results were compared, but no significant differences were demonstrated.

\section{INTRODUCTION}

La couverture végétale exerce un effet de filtre vis-à-vis des aérosols atmosphériques. Le dépôt se fait principalement sur les feuilles [4]. L'orientation des feuilles par rapport à la direction du vent n'a pas une grande influence [5]. Les feuilles étroites telles que celles des conifères ont un meilleur rendement de captation que les feuilles larges $[1,7]$.

Le Casuarina equisetifolia possède un feuillage qui ressemble à celui des conifères et il est très répandu sur tous les littoraux de la zone intertropicale. La mesure du plutonium déposé sur ses feuilles nous a permis de comparer le plutonium-239 atmosphérique de Mururoa et de Fangataufa, sites des expérimentations nucléaires en Polynésie française, à celui de l'ensemble de la zone intertropicale.

* Service mixte de contrôle biologique, BP 208, F 91311 Monthéry Cedex.

** Service mixte de sécurité radiologique; BP 208, F 91311 Montlhéry Cedex.

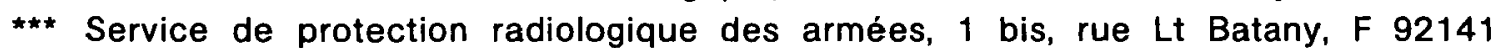
Clamart. 


\section{MATÉRIEL ET MÉTHODE}

\section{Casuarina equisetifolia}

Les noms vernaculaires de Casuarina equisetifolia Fost sont aito en Polynésie, filao dans l'Océan Indien et, plus communément, bois de fer. C'est un arbre très rameux de l'ordre des Casuarinales, famille des Casuarinacées. De loin, il ressemble à un pin. II s'en distingue par le fait que la tige ligneuse possède des rameaux aphylles : les feuilles sont remplacées par des gaines entourant les nœuds des rameaux sous formes d'écailles verticillées. Anatomiquement et physiologiquement, ce sont des feuilles qui, en première approximation, ressemblent à des aiguilles.

\section{Prélèvements}

Les prélèvements, d'un poids frais d'environ $250 \mathrm{~g}$, ont porté sur les feuilles des branches externes à une hauteur comprise entre 2 et $2,5 \mathrm{~m}$. L'étude a porté sur 84 échantillons dont 20 proviennent des sites d'expérimentations nucléaires de Mururoa et Fangataufa, 43 de Polynésie française hors sites expérimentaux (20 de l'archipel de la Société, 10 de l'archipel des Australes, 8 des Tuamotu-Gambier et 5 des Marquises) et 21 du reste de la zone intertropicale (3 de la côte ouest africaine Gabon et Sénégal), 6 des Antilles, 5 de Nouvelle-Calédonie et 7 de la Réunion. Chaque prélèvement est stocké en sac plastique soudé, tenu au réfrigérateur, voire au congélateur, pour éviter la putréfaction.

\section{Mesure}

Après pesée d'une partie aliquote de $250 \mathrm{~g}$, les échantillons sont calcinés à $500{ }^{\circ} \mathrm{C}$ avec montée en température programmée : montée à $300{ }^{\circ} \mathrm{C}$ en $2 \mathrm{~h}$, palier de $6 \mathrm{~h}$, montée à $500^{\circ} \mathrm{C}$ en $2 \mathrm{~h}$ et calcination pendant $14 \mathrm{~h}$. Après refroidissement, les cendres sont pesées et une partie aliquote de $1 \mathrm{~g}$ est soumise à l'attaque acide. L'attaque acide est réalisée par le mélange ternaire $\mathrm{HNO}_{3}, \mathrm{HF}$ et $\mathrm{HClO}_{4}$ dans la proportion 4/2/1. Dès l'obtention d'une phase liquide homogène, du plutonium 236 (37 mBq) est ajouté à titre de traceur. II permettra de calculer les teneurs en plutonium 239 en s'affranchissant de l'évaluation des pertes chimiques (le rendement chimique est estimé à $35,6 \pm 15,7 \%$ ) et du rendement de comptage.

Le plutonium est isolé par chromatographie sur résine anionique selon la méthode de TALVITIE [6]. Après fixation en phase HC I 9 N, l'uranium et le fer qui ont également été retenus sont éliminés par $\mathrm{HNO}_{3} 7,2 \mathrm{~N}$. Le plutonium est récupéré par élution en phase $\mathrm{HCl} 0,2 \mathrm{~N}$ en présence d'hydroxylamine, puis électro-déposé sur disque d'acier inox.

La mesure s'effectue par spectrométrie alpha à l'aide de détecteurs au silicium à zone partiellement désertée avec un montage NP, ou au moyen de chambres à grille. Le temps de comptage est à chaque 
fois de $15 \mathrm{~h}$. La quantité de plutonium 239 est calculée en rapportant la surface de son pic caractéristique à celle de celui correspondant au traceur.

Les valeurs, regroupées par zones, sont comparées par le test de Kruskal et Wallis. L'utilisation d'un test non paramétrique se justifie par l'impossibilité d'appliquer les conditions d'emploi d'un test paramétrique : normalité des distributions et égalité des variances. Les effectifs sont trop faibles pour que ces conditions soient vérifiables.

\section{RÉSULTATS}

Les résultats, exprimés en millibecquerels de plutonium 239 par gramme de cendre, sont présentés dans le tableau I, regroupés en zones : sites expérimentaux, archipel de la Société, Polynésie française hors sites et hors Société, reste de la zone intertropicale. Les valeurs indiquées ont toutes une probabilité d'être réelles supérieure ou égale à $50 \%$. Parmi elles, 19 ont une probabilité d'être réelles supérieure ou égale à $66 \%$ dont 6 supérieure ou égale à $97,5 \%$.

TABLEAU 1

Teneur en ${ }^{239} \mathrm{Pu}$ des feuilles de Casuarina equisetifolia (mBq/g de cendres)

\begin{tabular}{|c|c|c|c|}
\hline $\begin{array}{l}\text { Wururea } \\
\text { Fangataufa }\end{array}$ & $\begin{array}{l}\text { Archlpel } \\
\text { de la Societe }\end{array}$ & $\begin{array}{l}\text { Polynéste } \\
\text { Atrangalse }\end{array}$ & $\begin{array}{l}\text { Zzone } \\
\text { intertropicale }\end{array}$ \\
\hline $\begin{array}{l}0,5 \\
0,8 \\
1,0 \\
2,4 \\
0,5 \\
2,4 \\
9,1 \\
2,4 \\
2,9 \\
1,3 \\
0,5 \\
3,4 \\
0,8 \\
0,5 \\
0,5 \\
0,0 \\
1,2 \\
7,7 \\
0,5 \\
1,8\end{array}$ & $\begin{array}{l}2,4 \\
3,0 \\
0,7 \\
0,4 \\
4,9 \\
0,9 \\
0,2 \\
2,3 \\
1,8 \\
0,3 \\
0,2 \\
3,7 \\
1,4 \\
3,4 \\
1,1 \\
0,4 \\
2,4 \\
1,2 \\
1,2 \\
0,0\end{array}$ & $\begin{array}{r}1,5 \\
0,7 \\
2,3 \\
2,2 \\
1,0 \\
0,0 \\
0,4 \\
0,6 \\
0,0 \\
0,0 \\
1,1 \\
2,4 \\
0,0 \\
1,8 \\
11,6 \\
0,3 \\
7,3 \\
2,9 \\
6,5 \\
0,0 \\
0,0 \\
0,7 \\
1\end{array}$ & $\begin{array}{l}0,6 \\
0,3 \\
0,4 \\
4,6 \\
3,3 \\
1,0 \\
2,1 \\
0,3 \\
4,4 \\
0,9 \\
0,8 \\
0,2 \\
1,0 \\
0,6 \\
1,2 \\
0,6 \\
0,0 \\
0,7 \\
0,3 \\
0,5 \\
0,3\end{array}$ \\
\hline
\end{tabular}

* Hormis Mururoa, Fangataufa et l'archipel de la Société. 
Le test de Kruskal et Wallis appliqué aux résultats provenant des quatre zones permet d'accepter l'hypothèse d'égalité des quatre séries de résultats au degré de certitude $95 \%$ de façon hautement significative. En effet, l'hypothèse d'égalité est acceptée si la probabilité d'occurrence de l'hypothèse inverse est inférieure à $95 \%$; avec les résultats du tableau I, elle est égale à $59 \%$, donc très inférieure à 95 .

\section{DISCUSSION}

Les valeurs mesurées sont faibles. Elles s'étalent entre celle du bruit de fond de la mesure (indiqué par 0,0 ) et environ $12 \mathrm{mBq}$ de plutonium 239/g de cendres. Compte tenu du rapport d'incinération (environ $40 \%$ ), cette valeur maximale correspond à $0,005 \mathrm{mBq} / \mathrm{kg}$ de matière fraîche, expression la plus usuelle des résultats concernant l'environnement. Ces niveaux sont très bas. Parmi eux, les plus élevés ne proviennent pas tous des sites expérimentaux : quatre valeurs sont supérieures à $5 \mathrm{mBq} / \mathrm{g}$ de cendres, deux proviennent des sites et deux de l'archipel des Australes (îles de Tubaï et île de Rapa). L'île de Rapa, notamment, est située à environ $800 \mathrm{~km}$ au sud-ouest de Mururoa. Elle est trop loin et trop au sud des sites expérimentaux pour qu'une origine directe puisse être évoquée. Cette origine est plus vraisemblablement à rechercher dans les retombées mondiales.

Le test de Kruskal et Wallis n'a pas permis de différencier les valeurs provenant des sites expérimentaux de Mururoa et de Fangataufa de celles provenant d'ailleurs, en Polynésie française ou même dans le reste de la zone intertropicale.

A Fangataufa, l'essentiel du plutonium déposé sur la couronne émergée de l'atoll provient de la retombée consécutive à un essai atmosphérique exécuté à partir d'une barge en 1966. Pour augmenter les chances d'obtenir des mesures significatives, les points de prélèvement ont été volontairement choisis dans quelques endroits connus pour présenter les teneurs maximales en plutonium dans le sol ${ }^{1}$, à savoir de 1000 à $5000 \mathrm{~Bq} / \mathrm{kg}$ [2]. Cependant, les résultats de mesure sont peu élevés. Sachant que l'absorption racinaire du plutonium est généralement peu importante chez les végétaux, celui qui est mesuré provient essentiellement du dépôt foliaire. Sur cet atoll inhabité et sur lequel aucune activité expérimentale terrestre ne règne depuis plusieurs années, la remise en suspension qui ne peut être attribuée qu'aux seuls phénomènes météorologiques est, par conséquent, très faible.

A Mururoa, le bas niveau des mesures s'explique par le fait que nos prélèvements ont été pratiqués dans la zone de l'atoll réservée au logement des personnels du centre d'expérimentations ainsi qu'aux activités

\footnotetext{
1 A titre de comparaison, en ce qui concerne la teneur du sol en transuraniens [3], on rappelle que les niveaux d'action retenus à Enewetak pour la réutilisation d'anciens sites de tir par les populations ont été les suivantes:

- pour la résidence permanente: $<1480 \mathrm{~Bq} / \mathrm{kg}$.

- pour les travaux agricoles: $\quad<2960 \mathrm{~Bq} / \mathrm{kg}$,

- pour la cueillette: $\quad<5920 \mathrm{~Bq} / \mathrm{kg}$.
} 
industrielles et aéro-portuaires. Les résultats auraient, peut-être, été différents avec des prélèvements effectués sur des arbres poussant dans d'autres zones, notamment dans celle où, à l'époque des essais atmosphériques, ont été effectuées certaines expériences entraînant la dispersion de matière fissile. Cette zone a été assainie mais il y reste encore quelques "points chauds" dont la teneur des sols en plutonium est supérieure à celle trouvée à Fangataufa.

Dans les échantillons mesurés provenant des sites expérimentaux, l'origine du plutonium est double : le dépôt au sol dû à certains essais atmosphériques locaux et aux retombées dues aux essais atmosphériques mondiaux. Les résultats montrent que l'origine locale n'entraîne pas un accroissement significatif des valeurs.

\section{CONCLUSION}

La teneur du feuillage de Casuarina equisetifolia en plutonium 239 prélevé sur les sites des expérimentations nucléaires en Polynésie française ne présente pas de différence avec celle du feuillage prélevé dans l'archipel de la Société, dans le reste de la Polynésie française et dans d'autres endroits de la zone intertropicale. Ce résultat permet de penser que l'atmosphère, dans tous les lieux de prélèvement, présente la même concentration d'aérosols de ce radionucléide.

\section{BIBLIOGRAPHIE}

[1] BELOT Y., GAUTHIER D. - Mécanismes de transfert des particules microniques entre l'atmosphère et les plantes. Bull. Informations Scient. Techn. CEA, 1974, $N^{\circ} 191,67-73$.

[2] BOURLAT Y. - Communication personnelle, Service mixte de sécurité radiologique.

[3] ETATS-UNIS - Defense nuclear agency. The radiological cleanup of Enewetak atoll. Washington, D.C.: Defense nuclear Agency, 1981, p. 309.

[4] HOEFKEN K.D., GRAVENHORST G. - Deposition of atmospheric aerosol particles in beech and spruce forest. In : Deposition of atmospheric pollutants, 1981 (GEORGII H., PANKATH J., Eds.). Amsterdam : Kluwer, 1982, 191-194.

[5] KLEPPER B., CRAIG D.K. - Deposition of airborne particulates onto plant leaves. J. Environ. Qual, 1975, 4, 495-499.

[6] TALVITIE N.A. - Radiochemical determination of plutonium in environmental and biological samples by ion exchange. Anal. Chem., 1971, 43, 1827-1830.

[7] WATABE T., KAMADA H. - Airborne radionuclides onto pine needles collected in the vicinity of the nuclear power plant. J. Radiat. Res., 1984, 25, 140-149. 